\title{
Particle people compile data
}

\section{The latest compilation of particle physics data is a monument not merely to those who discover new particles but also to those who have improved the accuracy of what is known.}

THE complaint that there are simply too many material particles for any of them to justify the old adjective "fundamental" may seem, on the face of things, to be fully borne out by the latest compilation of properties by the Particle Data Group, a collaboration of 23 physicists which is an international outgrowth of the old Berkeley Particle Data Group on which responsibility for a critical review of available data used to rest. The new compilation, based on data which had become available by the end of 1983 , runs to 304 pages of the Reviews of Modern Physics for April this year (56, Sl-S304; 1984) and is likely to be many people's bible for years to come. It is also a model of how data should be compiled in a fast-moving field. The moral seems to be that the job is best done by people who are themselves practitioners.

In reality, the compilers have had to break with the traditions of their predecessors and now explain that they can no longer include references to all relevant publications in high-energy physics bearing on the properties of particles. The literature, they say, is growing by about 10 per cent a year, with the result that archival material must rest where it lies, in previous compilations, the most recent of which was two years ago. Laconically, however, the new list of particles includes (under "stable particles", if you please) the measured values of the masses of the $W$ and $Z$ particles first discovered just over a year ago at CERN, the European high-energy physics laboratory.

The classification of these particles (along with the photon) as stable is not as eccentric as it may seem. The criterion is that particles whose decay is accomplished only by the intervention of weak nuclear forces are counted as being stable. By the same test, a whole string of unstable mesons and baryons are thrown together in the same group.

To outsiders, perhaps the most surprising feature of these listings is the comparative speed with which it seems to have been possible to refine the accuracy of the masses of recently-discovered particles. Thus, among the group of three known leptons (electrons, $\mu$-mesons and $\tau$ mesons, each with negative or positive electric charge), the masses of the first two are understandably known to within one part in a million (after eight and four decades respectively), but even that of the $\tau$-meson is known to within 2 parts in 1,000 after less than a single decade. Among particle physicists, it must be tempting to hope for a further order of magnitude in the accuracy with which the mass of each particle is known with the passage of each decade. But this can only be a rule of thumb; the estimated mass of the first strange meson $(K)$, discovered more than thirty years ago, is hardly more precise (at one part in 50,000 ) than that of the $J / \psi$, just over a decade old and the first experimental proof that charmed quarks do indeed exist.

In the circumstances, it is something of a surprise that while the mass of the neutron is known (as it should be after all this time) to better than one part in a million, the lifetime of this particle against radioactive decay (into a proton, a negative electron and an antineutrino) is known only to within 2 per cent or thereabouts.

This is not, of course, the scandal that it may seem to be. The experimental difficulties of anything like a direct measurement of a decay whose products are among the most common particles in the real world, and - worse still - themselves stable, are bound to be formidable. But the practical importance of knowing the decay rate of neutrons is far from negligible even in such straightforward circumstances as the production of bursts of cosmic rays from the Sun.

On the lifetime of the proton, the new compilation of particle data is also necessarily unhelpful. The authors can only quote the steadily lengthening list of largely negative searches for evidence that protons do indeed decay, as the grand unified theories uniting electromagnetic, weak and strong forces suggest they should, into, say, electrons and photons. As is well-known, the lower limits on the lifetime determined experimentally are already less than the lifetime calculated by the simplest of the theories, according to this compilation by "at least one order of magnitude". Given that the first experimental data appeared only in 1981, and that nearly a score of substantial papers on the subject have since appeared, it cannot be much more than a year before the decay of the proton is observed, or the theories put in considerable difficulty.

Another of the still-fruitless searches reported in the compilation is that for the sixth quark, labelled top. By now, it seems thoroughly established that the particles called mesons consist simply of a quark and an antiquark, not necessarily of the same breed. In retrospect, it is clear that the most familiar mesons (the muon and pion) and the less massive of the two strange particles discovered in the late 1940s are made only of the three quarks called $u p$, down and strange. Charm was found at Stanford University a decade ago, and bottom soon afterwards, but top remains to be discovered.

This latest compilation of particle data has surprisingly little experimental evidence to report, all of it dating since 1981. And much of this is entirely negative - people have looked for particle resonances over a range of energies and have found nothing to suggest the existence of a top quark. But there is a handful of papers that appear to conclude that something happens at an energy of about $34 \mathrm{GeV}$ which could be the signature of the species called toponium, a particle built from the top quark and its antiquark. If that is the case, the proton-antiproton collider at CERN should find top any day now. Some would say that the missing quark should have been found already.

Even in this dry listing, the search for free quarks is more fun - but equally fruitless. In a listing of more than 250 experimental searches (each of which is solemnly accompanied by a number giving the number of "quark events" recorded), all but a few of the corresponding entries consist simply of the digit " 0 ". The experiments cover both searches for free quarks in accelerator experiments and those, based on the assumption that free quarks could be relatively massive and stable particles, in which people have sought to find quarks by taking vacuum cleaners to the ground beneath electrified security fences. The commentary in the compilation says that "of the several candidate cosmic ray events, one still enjoys the active advocacy of its discoverer"'.

Other particles have been sought and not found in great profusion. On the evidence of this listing, the magnetic monopole does not exist. From the exotic zoo of particles such as the Higgs boson (which should exist if only people knew where to look) and the tachyon (which entails time-reversal), the axion is singled out for special attention in this compilation, no doubt because of the recent popularity of these still unknown particles as plausible constituents of the missing mass in the Universe. But here again, for the time being at least, the experimentalists have drawn a blank.

John Maddox 